\title{
Development of Hydraulic Continuous Shifting Devices for the Host Machine of the Tunnel Boring Machine
}

\author{
Shuncheng Yang, Guo Zhou, Dengming Hu, Haiguo Zhang, Hongjian Gu
}

Shanghai Marine Equipment Research Institute of CSSC, Shanghai, China

Email address:

yshch001@163.com (Shuncheng Yang)

To cite this article:

Shuncheng Yang, Guo Zhou, Dengming Hu, Haiguo Zhang, Hongjian Gu. Development of Hydraulic Continuous Shifting Devices for the Host Machine of the Tunnel Boring Machine. Engineering and Applied Sciences. Vol. 5, No. 6, 2020, pp. 106-113. doi: 10.11648/j.eas.20200506.12

Received: June 6, 2020; Accepted: July 2, 2020; Published: November 19, 2020

\begin{abstract}
A powered continuous shifting device for the host machine of tunnel boring machine (TBM) is currently unavailable. Hydraulic power is the best choice of driving force source to develop the continuous shifting device which can carry 600-ton host machine in tunnel because of compact hydraulic subassemblies and efficient hydraulic control and transfer. According to the shape of the shield machine the cross section of the load-bearing platform is designed to be u-shaped and all subassemblies are placed in the left and right space under the arcuate crossbeam and four series wheels are arranged symmetrically on the outside. Hydraulic power is used to drive all hydraulic motors to make the device move and the driving wheel turn 90-degree, and push the piston in the corresponding oil cylinder, which fulfills functions such as lifting the host machine of the shield machine / TBM and turning all driven wheels 90 degrees etc. Polyurethane rubber with good comprehensive properties is used as the driving wheel coating materials to provide reliable driving force. The successful application of the device shows that its design principles and methods are feasible.
\end{abstract}

Keywords: The Host Machine of TBM, No-load Propulsion, Hydraulic Shift, Load-bearing Platform Design, Development and Application

\section{Introduction}

The tunnel boring machine typically falls into two types, namely shield machine and rock tunnel boring machine (TBM). It can be divided further into many other subtypes depending on geological conditions of the surrounding rock, shield forms, diameter, excavated section forms and tunnel horizontal-vertical angle. Tunneling technology has witnessed rapid development in the past decades, especially TBM, which has evolved into the most technically sophisticated tunnel boring machine that integrates multiple disciplines of mechanics, electricity, hydraulics, photics, control and material, and adapts to varied strata. It represents future direction of development in tunneling technology and is widely used. Current tunneling technology enables tunneling to be mechanized and standardized, greatly simplifying construction procedures with assembly line work. The development of China's economy requires unprecedented investment in infrastructure, a noticeable portion of which will go to tunneling projects. China has entered into a new era of underground space development, the demand for shield machine and TBM is growing at an annual rate of over one hundred [1-6]. Tunnel boring machine typically consists of the host machine of TBM, the most important component, and back up facilities. During tunneling (mainly subway tunnel) of the tunnel boring machine, the host machine of TBM usually has the following operations: originating horizontal shifting, no-load propulsion along the plus line (to heading face), no-load propulsion cross-station or $1 / 3,2 / 3$ or complete out-of-station. Current frequently used horizontal shifting technique is to use the hydraulic jack to lift the stand of the host machine of TBM on the manually laid steel rail, and the stand moves simultaneously; The conventional approach for no-load propulsion is to combine "pre-buried steel rails of the arc-shape guide table "with "no-load propulsion rack or rail clamping device", so the jack lifts no-load propulsion rack to conduct no-load propulsion operation. In summary, the above-mentioned horizontal shifting and no-load propulsion technique requires much preparation at the early stage and a high precision, delaying the overall progress. In addition, it is highly risky to construct the interface of the horizontal shifting and no-load propulsion, as the conventional approach requires 
one device complete transverse shifting and then another device complete no-load propulsion. There is no equipment or methods that can address both TBM horizontal shifting and no-load propulsion. The technical development in this regard doesn't keep up with the rapid development of the TBM technology. The gap in stark contrast has greatly impacted the construction efficiency and progress of the overall (subway) tunneling project.

A powered shifting device of the host machine of TBM is developed to achieve integrated operation of host machine horizontal shifting and no-load propulsion without welding or cutting the host machine housing. The new device combines horizontal shifting and no-load propulsion operation into one, reduces construction risks and improves the overall efficiency of the construction.

\section{Design Parameters and Goal of the Paper}

\subsection{Overview of the Host Machine of one Type of TBM, Major Load-bearing and Transport Body of the Designed Shifting Device}

The host machine of TBM typically consists of the cutter disc, fore shield, main drive, outer and inner extensible shield, support shield and rear shield. Due to the limitations of the machine's dimensions and weight, it is disassembled before going down the well. Once in the well, it is assembled on the shifting device. The machine includes a host machine of $600 \mathrm{t}$ and back up facilities of $400 t$, which travel with wheels on the rail. See Table 1 for weight distribution and size of host machine parts.

Table 1. Weight distribution and size of host machine parts.

\begin{tabular}{|c|c|c|c|c|c|c|}
\hline Parts & Cutter disc & Fore shield + main drive & Outer extensible shield & Inner extensible shield & Support shield & Rear shield+ erector etc \\
\hline Weight/t & 123 & $70+87=157$ & 19.2 & 18 & 197 & $25.6+60=85.6$ \\
\hline O. D. $/ \mathrm{mm}$ & 7032 & 6980 & 6960 & 6830 & 6880 & 6980 \\
\hline
\end{tabular}

\subsection{Operational Workflow of the Device}

The device has two operation stages: no-load propulsion origination and no-load cross-station. As there is no space in the well for the host machine and the device to make the steering, the change from horizontal transverse shifting to advancement along the plus line can only be achieved with the wheel reversing at the bottom of the device, but the host machine and the shifting device itself don't turn. No-load propulsion origination has the following working conditions: transverse shifting, wheel reversing, no-load propulsion along the plus line, unloading the host machine, device withdrawing, quick return to the originating well (so the back up trolley travels to connect with it). No-load propulsion cross-station has the following working conditions: loading the host machine, carrying the host machine and tugging back up trolley with the replacement wheel to the rear part of the next arc-shape guide table to dock with it, stop, unloading the host machine and the device withdrawing segment by segment.

\subsection{Requirements on Main Technical Parameters of the Device}

(1) Dimensions of the host machine of TBM: O. D. $\leq 8 \mathrm{~m}$, Length $\leq 14 \mathrm{~m}$;

(2) Weight of the host machine of TBM: $\leq 600 \mathrm{t}$;

(3) Cement flooring compression strength: $\leq 30 \mathrm{MPa}$;

(4) Advancing rate: $0.2 \sim 0.6 \mathrm{~m} / \mathrm{min}$;

(5) Retreating rate: $\sim 0.8 \mathrm{~m} / \mathrm{min}$;

(6) The distance from the bottom of the host machine to ground: Max. $370 \mathrm{~mm}$; the distance from the bottom of the shifting device to ground: $\leq 120 \mathrm{~mm}$.

\section{R\&D of the Device}

\subsection{Selecting the Right Type of Power}

Analysis is conducted according to the weight distribution and size of the host machine and the device' technical performance. First, as there is only limited place for the device and its parts, the dimensions of the designed and selected parts shall be very compact, its layout taken into the holistic consideration; secondly, the device travels very slowly, thus having no requirements on speed control; thirdly, as the weight of the host machine is not distributed evenly, the drive force provided by the driving wheels shall be free from its impact. There are three types of power: pneumatic, hydraulic and electric power. The pneumatic type is neglected due to limitations in power and noise harshness; the electric type is great in power, direct and efficient, and can be precisely controlled. It is easy to lay the wire and has good sealing, but it involves complex technologies for multiple sets of electric motors to simultaneously adapt to wide range load changes and speed regulation, not to mention, safety issues arising from use in the current environment. Other things being equal, the electric motor and its reducer and control elements are larger and more fragile than the hydraulic motor and its corresponding parts; the hydraulic parts are not only compact in size but have a high transfer efficiency and range of force. It is easy to regulate, control and use, particularly easy in speed regulation. Although the hydraulic motor requires hydraulic oil supply, electricity, oil tank and pump [7, 8], the hydraulic power is selected as the power source of driving wheels to meet the compactness and safety requirements, and the device is called the hydraulic shifting device.

\subsection{Structure, Components, Functions and Overall Layout of the Device}

\subsubsection{Structure, Components, Functions of the Device}

The device mainly consists of mechanical, hydraulic and electric control parts, as shown in Figure 1 (No.1 guard backer, 2 load-bearing platform, 3 oscillating steering motor, 4 mounting plate, 5 support skid, 6 round bar stringer, 7 driven wheels steering gears, 8 low driven wheels assembly, 9 portable fore-and-aft shifting guide wheel, 10 unloader, 11 
high driven wheels assembly, 12 lifting assembly holder, 13 transverse shifting guide mechanism, 14 driving wheels assembly), of which:

(1) The mechanical part mainly consists of the load-bearing platform, low driven wheels assembly, high driven wheels assembly, driving wheels assembly, transverse shifting guide wheel, portable fore-and-aft shifting guide wheel, driven wheels steering gears, unloader (the host machine), lifting assembly holder and trailer trolley.

(2) The hydraulic part mainly consists of:

a. Hydraulic supply: electric motor pump units, including variable plunger piston pump units and vane pump units;

b. Power actuating units: travel drive motor, oscillating steering motor, leg cylinder, transverse shifting guide wheel cylinder, wheel pressure cylinder, driven wheels steering cylinder, and shield unloading cylinder;

c. Control valve units: solenoid directional valve, hand-operated direction valve, speed-regulating valve, one-way valve, safety valve units, pressure-relief valve, sequence valve, proportional direction valve and multi-level overflow valve etc.

d. Hydraulic piping accessories etc.

(3) The electric control part mainly consists of electro-hydraulic console (including electric control operation and hydraulic control manual operation), electrical junction box webcam, headlight, distribution box and quick-coupled adaptor.

The load-bearing platform is the framework of the whole device, of which the mounting plate is used to mount various assemblies, round bar stringer only for mounting low driven wheels assembly and the support skid for mounting the host machine of TBM. Some devices or assemblies is connected to the corresponding power actuating units to fulfill relevant functions; driving wheels assembly includes the oscillating steering motor, wheel pressure cylinder and travel drive motor; transverse shifting guide wheel includes transverse shifting guide wheel cylinder; driven wheels steering gears includes driven wheels steering cylinder; unloader (the host machine) includes shield unloading cylinder; the lifting assembly holder includes the leg cylinder; the naming of the cylinder reflects its functions. Control valve units concentrate on a manifold block of the valve unit so as to hydraulically control power actuating units; electro-hydraulic console, electrical junction box and hydraulic piping accessories are used to control the whole device electrically and hydraulically.

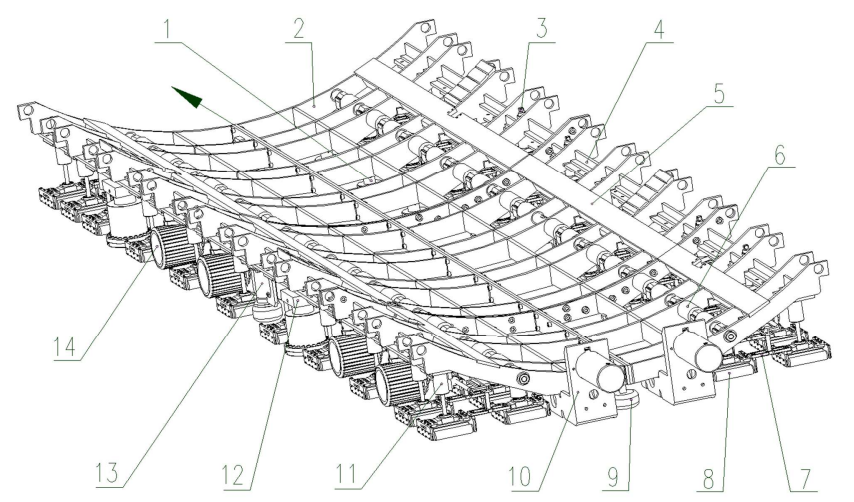

Figure 1. Structure, composition and component layout of the shifting device.

\subsubsection{Layout of Major Components of the Device}

The device is segmented the same way as the load-bearing platform segmentation: fore segment, middle segment and rear segment. As indicated in Figure 1, the direction of the arrow indicates the fore segment of the device, i.e. the advancing direction. Two lines of wheels are arranged under the mounting plates on the outer left side and right sides of the fore segment: high driven wheels assembly, driving wheels assembly, transverse shifting guide wheel and lifting assembly holder, while two lines of low driven wheels assemblies are arranged under the round bar stringer on the inner left side and right sides of the fore segment. Driven wheels steering gears are arranged symmetrically under the support plate of each segment on the left and right side. As needed; the driving wheels assembly is arranged on each segment to operate independently. Considering that the weight of the host machine is distributed mainly in the fore segment instead of the rear segment, two of four sets of lifting assembly holders are arranged in the front of the fore segment (near the fore shield and cutter disc), the other two sets are arranged in the end of the middle segment (near the support shield). The bottoms of these four cylinders are suspended from the ground at a height of $70 \mathrm{~mm}$, upper piston top fixed to the holder. Two sets of Transverse shifting guide mechanism are arranged in the middle segment, almost the middle of the whole device; 2 sets of portable fore-and-aft guide wheel are arranged in the longitudinal center, near the fore part of the fore segment and the rear part of the rear segment, and the unloader is hanged at the rear part; the webcam and two headlights are arranged in the fore part, which can be installed or dismantled as needed.

The electro-hydraulic console, electrical junction box, oil tank and accessories, electric motor pump units and the manifold block of valve units concentrates on a frame, which connects to a trailer trolley to form a electro-hydraulic console trolley, as shown in Figure 2 (No. 1 electro-hydraulic console, 2 electrical junction box, 3 oil tank and accessories, 4 electric motor pump units, 5 the manifold block of valve units, 6 trailer trolley. The relative position between the trolley and the load-bearing platform depends on the shifting direction of the device: during the transverse shifting, the trolley is articulated to the side of the rear of the device; during the shifting along the plus line (fore-and-aft shifting), the trolley turns $180^{\circ}$ to connect with the rear of the load-bearing platform. In both cases, the trolley is hauled forward by the load-bearing platform; the device is controlled from the manual operated panel on the electro-hydraulic console. In addition, the three segments of the device are connected with high strength screws, which can be detached or connected. The hydraulic piping is arranged on the outer left and right sides of the platform; the segments are connected with quick-coupled adaptor that can be easily connected or detached. When detached, each segment can work independently of each other; via several $15 \mathrm{~m}$ hoses, pressure hydraulic oil on the trolley can be delivered to each hydraulically powered actuating units or returned to the oil tank. 


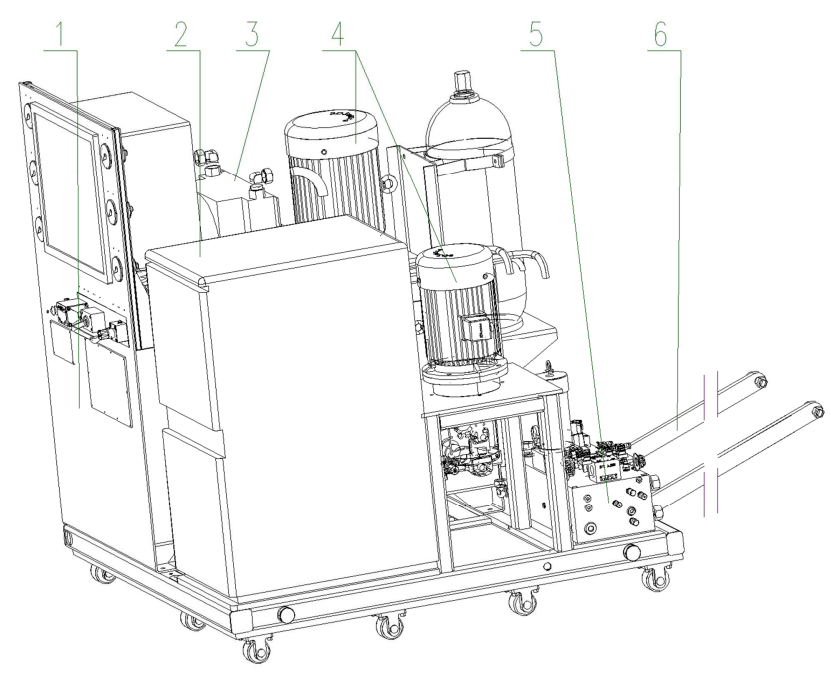

Figure 2. Structure and composition of the electro-hydraulic console trolley.

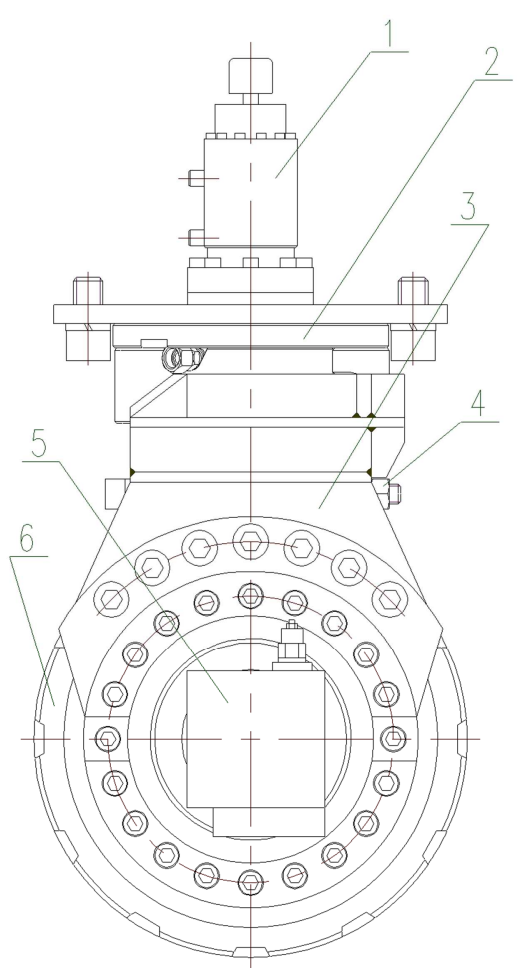

Figure 3. Structure and composition of the driving wheel assembly.

\subsection{Functional Principle of the Device}

All the preparation is ready (including reserve guide groove at the center of the ground), the starting location of the wheels is in the fore-and-aft direction of the device, all the pistons in the cylinder retracted, i.e. the stroke is zero. First start two electric motor pump units (Max. pressure of variable plunger piston pump units is $25 \mathrm{MPa}$, max. pressure of vane pump units is $17.5 \mathrm{MPa}$, the former is for use of oil cylinder and oscillating steering motor while the latter is for travel drive motor), meanwhile start the wind cooler motor, which will automatically heat if the hydraulic oil temperature is below $25^{\circ} \mathrm{C}$, and stop heating if above $50^{\circ} \mathrm{C}$. Two pressure oil supplies are ready.
Transverse shifting:

Charge the hydraulic oil to the leg cylinder in the four sets of lifting assembly holders, the piston of the leg cylinder unmoved, cylinder barrel compressing internal spring, the bottom of the cylinder barrel extending downwards till it touches the ground, piston holder going up too, which propels the load-bearing platform to lift all the driven wheels off ground (no pressure on the driving wheels); then charge the hydraulic oil to the driven wheels steering cylinder and oscillating steering motor, under the action of steering gears of driven wheels, all the driven wheels turns $90^{\circ}$; the cylinder rod of the oscillating steering motor propels the cylinder rod of wheel pressure cylinder to turn (no pressure on the wheel pressure cylinder); the lower end of its cylinder rod is secured to the combined holder of driving wheels assembly by the long screw to propel the combined holder to turn the compound wheels for $90^{\circ}$, as shown in Figure 3 (No. 1 oscillating steering motor; 2 wheel pressure cylinder; 3 combined holder; 4 long screw; 5 travel drive motor; 6 compound wheel). Note one end of the compound wheel is connected to the travel drive motor, the other end directly connected to the rolling bearing embedded in the combined holder. After it is over, relieve pressure from the leg cylinder, the spring in the leg cylinder reset, its cylinder barrel retracted and suspended. Due to gravity, the platform goes down till the wheels touch the ground and withstand the load, and then charge hydraulic oil to wheel pressure cylinder, under the action of the cylinder rod, the platform doesn't move if the load-bearing platform carries heavy load no less than the total applied force on the wheel pressure cylinder, and the pressure of the compound wheel against the ground is equal to the applied force. Otherwise the platform will be lifted. Next according to the relative position of transverse guide wheel to the guide groove on the ground, start travel drive motor to advance or retreat so that the two transverse guide wheels face directly the guide groove on the ground, and stop so its wheels extends into the groove under the oil charge action of the transverse guide wheel; after it is over, start the travel drive motor, so the device tows the electro-hydraulic console trolley to advance transversely; when the travel drive motor crosses the plus line guide groove with the block in the groove and face the fore-and-aft middle line of the device, close it, stop and retract the transverse guide wheel under reverse action of the transverse wheel cylinder, remove the block in the groove, disconnect the electro-hydraulic console trolley from the platform, thus completing the transverse shifting.

Main line shifting (Longitudinal shifting):

Following the above, first retract and lift the driving wheels assembly under the reverse oil charge action of the wheel pressure cylinder, unload the pressure (driving wheels unstressed), pressurize the leg cylinder, lift driven wheels off ground, fill oil to driven wheels steering cylinder and oscillating steering motor, and make driven wheels and driving wheels turn $90^{\circ}$ towards the fore-and-aft direction under the action of corresponding mechanisms. Then sink two sets of portable fore-and-aft guide wheels towards the guide groove on the ground respectively, and put them in the 
designated groove in the front and back of the device. Charge oil to and pressurize wheel pressure cylinder, start travel drive motor, stop after advancing for $5 \mathrm{~m}$, turn the electro-hydraulic console trolley $180^{\circ}$, connect it to the rear of the device, and the device will haul it to move forward. (If it slides too fast or can hardly stop going downhill, try quick oil charge action of transverse guide wheel cylinder, and the guide wheels will quickly extend and the back presses firmly against the cement ground to brake.) The device continues advancing to the rear of arc-shape guide table, dock, stop and relieve pressure from the wheel pressure cylinder, charge oil reversely to and lift driving wheels assembly, and the shifting along the plus line is complete.

Unload the host machine:

Weld the block frame on the reserve steel plate on the ground of the rear of the device to store the unloader, charge the hydraulic oil to two shield-unloading cylinders on top of the unloader, the front part of the cylinder rod lifting the host machine, which slides on two support skids of the load-bearing platform and enters the arc-shape guide table. After the rear of load-bearing platform has space, the unloader moves to take up the space; its bottom secured to the platform crossbeam, the shield unloading cylinder continues to lift the host machine, the unloader inching forward till the host machine is completely away from the platform.

Final retreating:

The flame cut off the welded block frame on the ground. Pressurize driving wheels, start travel drive motor backwards, and the device retreats to stop at the T-crossing. The wheels turn and shift transversely to the originating point. The device can also be carried back on a fork-lift truck at a faster speed. During retreating, the platform pushes the electro-hydraulic console trolley forward.

\subsection{Design of the Device Components}

The design of the device mainly involves the design of mechanical, electric control and hydraulic parts. The electric control design is mainly based on the operational sequence and safety requirements of the hydraulic actuating units. Electrical parts are selected according to strict logic and can control and protect hydraulic actuating units. The design principle of the whole hydraulic system corresponds to the functional principles of the above device. The design process involves calculation of relevant liquid parameters, selection of hydraulic units and design of dedicated units according to the design input, mechanical design requirements and hydraulic design standards of the whole device, which can be regarded as the regular design worth no further explanation. The design of several main components is introduced as follows.

\subsubsection{Design of Load-bearing Platform}

Load-bearing platform is the framework of the whole device, which is vital to the safety and reliability of the device. The convenience for transportation and lifting shall also be taken into overall consideration.

Load-bearing platform features $U$ shaped welded bracket. It is $13.05 \mathrm{~m}$ in total length, similar to that of the shield body, and $4.5 \mathrm{~m}$ in width, making the best use of transverse space of the no-load propulsion passage. Considering width of the originating well and convenience for transport, the load-bearing platform is designed to be a six segment assembly which can be transported segment by segment. When in use, it can be divided into the fore segment, middle segment and rear segment. The three segments are secured to each other by regular bolts and hexagon head articulated bolts. The gravity of the host machine is distributed all over the platform for each wheel (its layout already introduced above) to carry. To increase its stiffness and bending modulus, the stiffened plates are mounted on the mounting plate and below the support skid.

Due to uneven distribution of the weight of the host machine in the fore segment, the three segments are designed into different length to match the load capacity. UG drawing software is used to build 3D modeling for the platform, ANSYS and ABAQUS are used to calculate the strength of the platform. Conduct simulation calculation of various operating conditions such as lifting, horizontal transverse shifting + no-load propulsion along the plus line and reversing. Under each operating condition, the wheels are stressed differently, thus changing the stress of the platform. During the calculation, analyze half of the model according to the symmetry of the device [9-11]. The calculation analysis is only briefly mentioned here in the paper, further elaboration can be found in other relevant articles.

Comparing the maximum distortion and stress and relevant parts under the above three operating conditions, the maximum stress and deformation occurs at the fore segment, near the place where the fore shield is installed. In addition, the maximum equivalent stress on the support skid is 446.6 $\mathrm{MPa}$ (also the contact crushing stress), the maximum spring deformation $37.16 \mathrm{~mm}$ (The upper part of driven wheels are installed with disc springs). Meanwhile the maximum equivalent stress of the stiffener at the bottom of the support skid is $314.9 \mathrm{MPa}$ while other parts have smaller stress approximately $200 \mathrm{MPa}$. In light of stress distribution, different materials are selected for different parts. As the platform features a welded structure, the weldability and between different materials and welding process shall be taken into the overall consideration. It is decided that the support skid uses low alloy and high strength fabricated steel [12]: Q690 C (GB/T1591-2008), the stiffened plate and cross beam under the support skid uses Q460D (GB/T1591-2008), other parts use Q345 (GB/T1591-2008). The load-bearing platform weighs 27 tons in total.

\subsubsection{Design of Driving Wheels Assembly}

(1) Structure, components and operating principle of the driving wheels assembly

See Figure 3 for the structure and composition (No. 1 oscillating steering motor; 2 wheel pressure cylinder; 3 the combined holder; 4 long screw; 5 travel drive motor; 6 compound wheel). The guide groove at the top of the cylinder rod of the wheel pressure cylinder is connected to the oscillating steering motor via the flat key, and the bottom of 
the cylinder rod is secured to the combined holder via the long screw. The hydraulic force in the oil cylinder drives the piston rod up and down, and the torque of the oscillating steering motor rotates it, which respectively propels the combined holder to move up and down or rotate. Travel drive motor is fastened to the combined holder at one end, and fastened to reduction gears of the external compound wheel (driving wheels) at the other end. The compound wheel is directly connected to the combined holder via the rolling bearing at one end; the top of the combined holder has a drum wrapped on the outer circle of wheel pressure cylinder barrel. It can slide up and down and rotate to prevent the cylinder rod from directly bearing the transverse force, thus protecting the seal ring. As such, the oscillating steering motor propels the driving wheels to turn $90^{\circ}$, and the wheel pressure cylinder can make driving wheels slide up and down and press firmly against the ground to produce the required friction as driving force of the device. The wheel pressure cylinder has a piston stroke of $30 \mathrm{~mm}$, the maximum stress of $180 \mathrm{kN}$, i.e. the maximum pressure against the ground of $180 \mathrm{kN}$.

(2) The design and machining technique of compound wheels (driving wheels)

The design of compound wheels is the most important in the design of driving wheels assembly, which determines the reliability of producing driving force of the device and service life. Compound wheels consist of steel wheels and wrapping layers. In terms of load distribution and stress, the contact stress between steel wheels and cement ground (approx. calculated by herz stress equation,) far exceeds the pressure resistance of the cement ground, which damages the ground. After repeated rounds of calculation, polyurethane (rubber or elastic body PUE), a non-metallic material with very small elastic modulus E, is selected as the steel wire wrapping. It is selected because polyurethane is a high polymer elastic material between rubber and plastics sharing essential properties of both. It has a hardness range of Shore A65 A98, a temperature range of $-50^{\circ} \mathrm{C} \sim 120^{\circ} \mathrm{C}$, with a rather high strength, elasticity, outstanding antiwear, and oil resistivity and hydrolysis resistivity. In addition, it has a mechanical strength 2-3 times of that of natural rubber and antiwear 5-10 times of natural rubber, and the binding property with metal is good too. In sum, it has good overall performance [13, 14]; these properties fully meet the onsite environmental and operational requirements of driving wheels. More importantly, although the frictional behavior of elastic body of polyurethane differs significantly from that of metallic materials, i.e. its kinetic friction coefficient is greater than static friction coefficient, and the difference between them increases with speed within a certain speed range, driving wheels can benefit greatly from its anti-wear, high friction coefficient, and the fact that the friction coefficient increases in case of high temperature, and friction coefficient against the steel can easily be as high as 0.46 [15]. As the elastic body of polyurethane is soft, highly elastic and poor in heat conduction, it is difficult to cut and process it to the required shapes, dimensions and surface roughness. Instead, it is processed by being melted to liquid and casting. Sophisticated moulds are designed for the purpose to cast compound wheels at one go without any other processing.

\subsubsection{Design of Driven Wheels Assembly}

The design target of driven wheels assembly is: (1) can withstand 20 tons; (2) can adapt to slopes of about $5^{\circ}$ and $\pm 15 \mathrm{~mm}$ roughness; (3) contact stress of the wheel against the ground no greater than pressure strength of cement ground (4) can rotate around its own axis to achieve steering.

Driven wheels assembly consists of high driven wheels assembly and low driven wheel assembly, its structure and composition shown in Figure 4 (No.: 1 semi-circular blind flange, 2 disc spring, 3 articulation bearing, 4 nylon wheels, 5 force bearing coupled pole, 6 guide pressure cylinder, 7 thread pin, 8 long force bearing coupled pole, 9 flat guide pressure cylinder), the figure on the left and right shows the low driven wheels assembly and high driven wheels assembly.
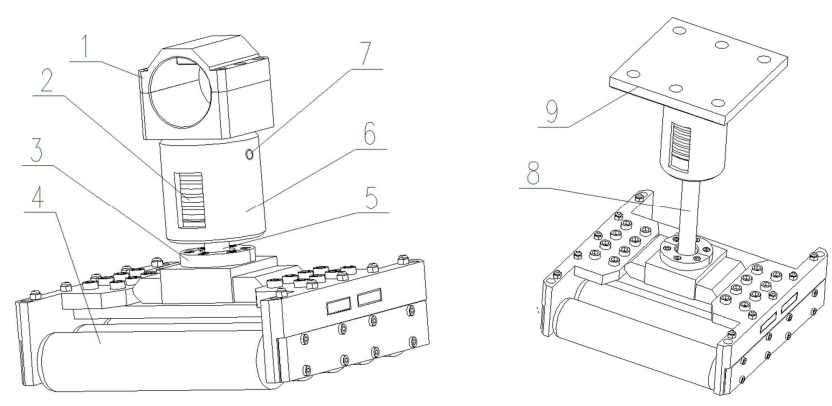

Figure 4. Structure and composition of the driven wheel assembly.

The difference between high driven wheels assembly and low high driven wheels assembly lies solely in the shape of the upper connection and the length of the force bearing coupled pole in the middle. Articulation bearing connects the upper and lower part; nylon wheels can rotate $360^{\circ}$ around the bearing center line, or bob up and down $\pm 5^{\circ}$ of the horizontal plane; the force bearing coupled pole has a guide groove with an axial length of $35 \mathrm{~mm}$, the top of which is inserted into a guide hole at the top of the guide pressure cylinder with a 35 $\mathrm{mm}$ gap reserved; disc springs are arranged in series in the circular steps at the top of the force bearing coupled pole; the sides of circular steps can slide up and down in the inner wall of the guide pressure cylinder; the thread pin goes through the guide groove at the top of force bearing coupled pole, and is fastened to the outer wall of the guide pressure cylinder, to prevent the force bearing coupled pole and its lower parts from falling when the device is lifted. In this way, the driven wheels assembly can adapt to a certain degree of inclination and denivellation while rotating. Nylon is selected as the wheels materials due to its high strength, low density, smooth surface and low friction coefficient.

\subsection{Tests for Major Components and Factory Acceptance of the Device}

Test of major components mainly consists of load test of the load-bearing platform, driving wheels assembly, driven wheels assembly. First conduct load test of the driving wheels 
assembly and driven wheels assembly. On the hydraulic load test bench, place the driving wheels on leveled cement ground cast as the tunnel ground, load 20 tons, the compression of wheel bottom wrapping layer (tire) is $4 \mathrm{~mm}$ (the original thickness is $30 \mathrm{~mm}$ ). A set of small device is specially designed to measure sliding friction coefficient of the tire. It can measure friction coefficient indirectly by making cement ground turn without the wheel turning (the minimum value shall be no less than 0.4 ). The loading range of driven wheels is 20 30 tons. Nylon wheels don't have noticeable press deformation, maximum deformation $2 \mathrm{~mm}$. Driving and driven wheels have small deformation in the wheel stand, while the wheel has the most deformation. The test demonstrates these two components meet design requirements The platform is tested with the rear segment ( 3 meter long) carrying 165 tons of actual loading (support shield framework), and moving forward and backward driven only by the hydraulic drive. As it is representative, its good test results are applied to the whole platform. Factory acceptance test of the device is mainly to conduct all the operations on an empty ground according to the operating procedures and requirements at no-load, and demonstrate that functions such as transverse shifting, fore-and-aft shifting, lifting, retreating meet requirements.

\section{Onsite Application and Actual Effect of the Device}

After the factory acceptance test, the device is first used in Yanjiashan Metro Station of Line 8 in Qingdao. The boring machine is TBM, the host machine 640 tons, the maximum diameter at the bottom $6980 \mathrm{~mm}$. Brackets of various thickness is prepared so that several important parts come into contact with the support skid of the load-bearing platform. The host machine of TBM is assembled on the platform: the device shifts in a fore-and-aft direction, so the support shield on the platform faces directly where the crane boom falls at the opening (because the support shield is too heavy, and the crane boom is relative short); first lower the support shield (197 tons), retreat to the originating position, then lower the fore shield, main drive and inner and outer extensible shield, finish the connection, lower the cutter disc, last lower the rear shield, finish the assembly. All the wheels turns $90^{\circ}$ and shift $45 \mathrm{~m}$ in the transverse direction at a rate of $0.6 \mathrm{~m} / \mathrm{min}$; once in the plus line position, all the wheels turn $90^{\circ}$; after the erector is mounted on the rear shield, advance $150 \mathrm{~m}$ along the plus line to the guide table; unload the host machine, and the cutter disc meets the heading face. The device returns to the originating position, and is lifted out of the opening. The project is finished 20 days ahead of schedule in this way compared with conventional methods, which sets a record and marks the first success in application.

The device turns out to work well in real-life applications, and the performance meets the design technical requirements and achieve projected research target.

\section{Issues and Improvements Measures}

Two issues emerge in the onsite application. One is that driving wheels assembly is not that well adapted to the ground To be specific, the $30 \mathrm{~mm}$ piston stroke of wheel pressure cylinder is too short, so it is easy for the wheels to slip, and the requirements for the road planeness is as high as $\pm 15 \mathrm{~mm}$; the second is that the corresponding guide wheel is not useful during transverse shifting and fore-and-aft shifting, especially big-turn. A cylinder is needed on the side to rectify the deviation. The following measures are proposed for the design of next device: to increase the piston stroke of wheel pressure cylinder to at least $60 \mathrm{~mm}$ or as long as possible within the allowable space. Meanwhile increase the quantity of driving wheels assemblies, have enough back up power, cancel the guide wheel and use the speed difference between driving wheels on the two sides to achieve steering.

\section{Conclusion}

The successful application of the device and inspection of the device after use proves that the designs according to design input is basically right and proper. It proves the feasibility to use hydraulic power to shift the host machine of TBM in terms of the principles and methods. Evidence shows that the successful development of this device has filled the blank in the technology across the globe. The application in the subway tunnel will create more demands for the new device.

\section{References}

[1] Zeng Xiangrong, Zhang Mingming, Liu Zaizheng etc. Design of hydraulic bracing systems in the propulsion of double shield hard rock tunnel boring machine [J]. Railway construction technology, 2017 (10): 34-37.

[2] Wang Mengshu. Technical state, existing issues and development ideas of shield machine and TBM tunneling [J]. Tunnel construction, 2014 (3): 180-181.

[3] Yukinori Koyama. The state and technology of shield tunneling method in Japan $[\mathrm{J}]$. Tunneling and Underground Space Technology, 2003 (18): 89-103.

[4] Long Zhiyang, Guo Xiaoxian. Development and application of full face tunneling machine [J]. Tunneling technology, 2017, 38 (5): 7-13.

[5] Qian Qihu, Li Zhaobu, Fu Deming, Application and outlook of TBM in China's underground engineering project [J]. Underground space, 2002, 22 (1): 1-11.

[6] Guo Shanyun. Development and Application of shield technology [J] Construction mechanization, 2009 (07): 24-27.

[7] Zhang Hongjia, Huang Yi, Hydraulic drive [M]. Beijing: China Machine Press, 1997.

[8] Ruan Yi, Chen Boshi, Electric drive automatic control systemmotion control system [M]. Beijing: China Machine Press, 2013.

[9] Shi Yiping. Detailed explanation of ABAQUS finite element analysis examples [M]. Beijing: Mechanical Industry Press, 2007. 
[10] Ding Yuan. From introduction to mastery of ABAQUS2018 finite element analysis [M]. Beijing: Tsinghua university press, 2019.

[11] Xu Jingjing. ANSYS13.0 Workbench Numerical simulation technology [M]. Beijing: China water power press, 2012.

[12] GB/T1591-2008, Low alloy high strength structural steel [S].

[13] Yu Yongming, Abrasive machining of polyurethane friction disk [J]. Diamond and grinding medium and tools, 2005 (4): 62-64.

[14] Jiang Jiajian, Xu Xunwei. Study on abrasive planing of polyurethane top roller [J]. Equipment manufacturing technology, 2010 (4): 173-174.

[15] Lin Fuyan, Ma Dongsheng, Ma Xiangdong, Study on frictional properties of the frictional gasket material on polyurethane elastic mass [J] Lubrication and Sealing, 2000 (2): 23-24, 60. 